in basal and mid short axis. The images were analysed by blinded readers to assess the reproducibility of the 12 segment myocardial segmentation approach.

Patients were recruited from the Rheumatology department which is a regional centre for myositis. Patients with cardiac symptoms or elevated troponin I were recruited. Recruits underwent CMR with tissue mapping and late gadolinium enhancement at the first visit with 2 subsequent non-contrast mapping studies followed by a final gadolinium enhanced CMR at 12 months.

All patient scans were performed on the same 1.5T scanner and analysed by the same reader. In order to mitigate for bias a second reader, blinded to the clinical details including the troponin, repeated the analysis on $50 \%$ of studies. The T1 and T2 were calculated for 12 segments in each scan.

Results Reproducibility of a 12 segment model for analysing tissue mapping sequences is excellent with a coefficient of variability of $1.4 \%$ for $\mathrm{T} 1 \%$ and $2.6 \%$ for T2. Myositis patients with elevated troponin I had a significantly higher mean T1 and T2 than healthy volunteers $(\mathrm{p}<0.0001$ both). Myositis patients with elevated troponin $\mathrm{i}$ have a significantly higher T1 and T2 than myositis patients with normal troponin I $(\mathrm{p}<0.01$ both). Patients with myositis and a negative troponin I have a significantly higher $\mathrm{T} 1$ and $\mathrm{T} 2$ than healthy volunteers (T1 $\mathrm{p}<0.01, \mathrm{~T} 2 \mathrm{p}<0.05)$. There is a significant reduction in T1 and T2 values in patients with treatment over 8 months $(\mathrm{p}<0.05$ both).

Conclusions A twelve segment model for myocardial T1 and T2 assessment is highly reproducible which is important in this disease process as there can be either focal or diffuse myocardial involvement. T1 and T2 are strongly associated with troponin I positivity with both likely to be a reflection of active inflammation in this disease process and improve with immunesuppression therapy fairly quickly.

\section{TRAIN THE AI LIKE A HUMAN OBSERVER: DEEP LEARNING WITH VISUALISATION AND GUIDANCE ON ATTENTION IN CARDIAC T1 MAPPING}

Qiang Zhang, Konrad Werys, Elena Lukaschuk, Iulia Popescu, Evan Hann, Stefan Neubauer, Vanessa M Ferreira, Stefan K Piechnik. OCMR, University of Oxford Centre for Clinical Magnetic Resonance Research

\subsection{6/heartjnl-2019-BSCMR.9}

Background Artificial intelligence (AI) is increasingly used in diagnostic imaging. Deep convolutional neural networks (CNN) are able to learn from datasets presented to them, and then provide independent interpretations on new cases, but often without traceability of how they came to the conclusions. Such 'black box' behaviour is not desirable for clinical use. We propose the concepts of visualising and guiding the AI attention in an application to artefact detection in cardiac T1-mapping - a critical quality assurance step for clinicallyrobust T1 determinations.

Method We utilise the emerging AI attention visualisation. This serves as an 'eye tracker' and reveals where the neural network 'looks' when scoring artefacts in T1 mapping, and adds an essential accountability aspect to the CNN by producing additional evidence to validate the decision making process. Beyond simply observing the perception, we developed a technique to provide direct guidance on the attention of the $\mathrm{CNN}$, by telling the machine which region to look at, very similar to training a human observer.

Results We demonstrate an application in automated T1 mapping artefact detection of the 6 AHA segments in mid-ventricular slices (figure 1a). The AI 'eye tracker' detected an illtrained CNN paying attention to features not desired for the assigned tasks (figure 1b). A well-trained CNN learned from the training data to pay attention to the corresponding

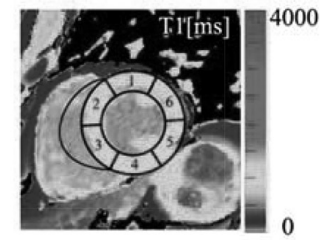

(a) AHA segmental model

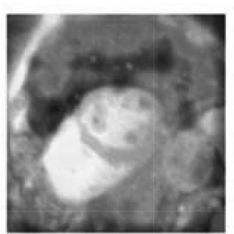

(b) Attention of an ill-trained $\mathrm{CNN}$
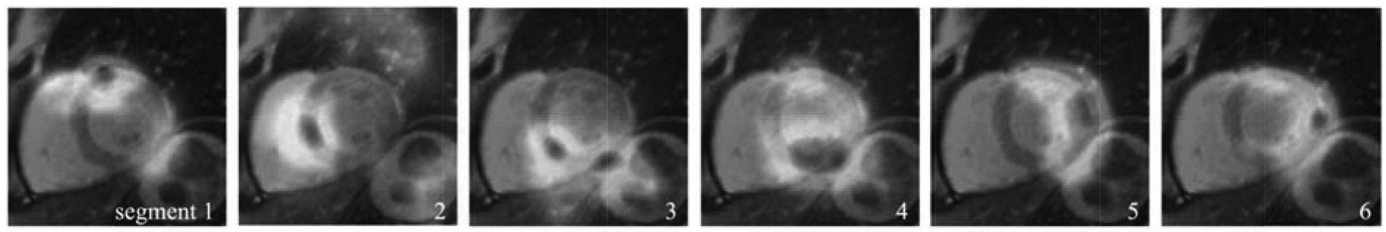

(c) Attention of a well-trained $\mathrm{CNN}$ when detecting artefacts in 6 segments
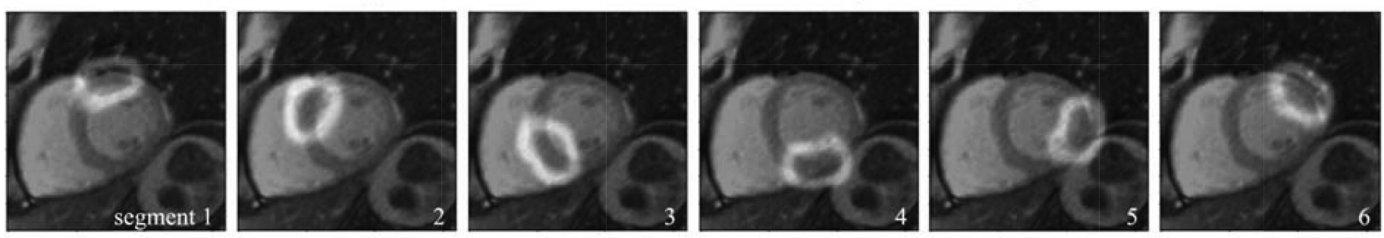

(d) $\mathrm{CNN}$ trained with proposed attention guidence technique

Abstract 9 Figure 1 Attention visualisation and guidance in detecting T1 mapping artefacts in the 6 AHA segments (a), which reveals that (b) an ill-trained CNN looked at the features irrelevant to the tasks, (c) a well-trained CNN highlighted the segments but with distraction by other image features, and (d) with attention guidance in training the CNN highlighted the segments more accurately 
myocardial segments for detecting artefacts, but with indicating distractions leading to suboptimal accuracy (figure 1c). A $\mathrm{CNN}$ trained with additional guidance on attention is shown to pay the desired attention to the right structures and avoids distractions (figure 1d).

Conclusion CNN designed with support of attention visualisation, and trained with guidance on attention can lead to significantly more transparent and accountable AI use in clinical practice.

\section{INTER-FIELD STRENGTH AGREEMENT OF MYOCARDIAL STRAIN MEASUREMENTS USING TISSUE TRACKING CARDIOVASCULAR MAGNETIC RESONANCE IMAGING}

S Ayton, L Athithan, KS Parke, JV Wormleighton, C Budgeon, GP McCann, GS Gulsin. Department of Cardiovascular Sciences, University of Leicester, and NIHR Leicester Biomedical Research Centre, Glenfield Hospital, Leicester, UK

\subsection{6/heartjnl-2019-BSCMR.10}

Funding acknowledgement The participants included in this study were recruited as part of a larger study funded by the National Institute for Health Research Career Development Fellowship (CDF 2014-07-045).

Background The agreement of left ventricular (LV) strain measurements using cardiovascular magnetic resonance (CMR) tissue tracking at different field strengths is unknown. We compared inter-field strength agreement of systolic and diastolic strain/strain rates at $1.5 \mathrm{~T}$ and $3 \mathrm{~T}$.

Methods We prospectively enrolled 17 healthy volunteers and 3 participants with diabetes but no cardiovascular disease, from a diet and exercise intervention study. Subjects underwent CMR balanced steady state free precession cine imaging with retrospective ECG gating at $3 \mathrm{~T}$ followed by $1.5 \mathrm{~T}$ on the same day. Acquisition voxel size was $1.66 \times 1.33 \times$ $8 \mathrm{~mm}$ and $1.90 \times 1.52 \times 8 \mathrm{~mm}$, respectively, temporal resolution $48 \mathrm{~ms}$ and $38 \mathrm{~ms}$, TR 3.44 and 2.76 and TE 1.51 and 1.15. Acquisitions were reconstructed to 30 phases. Short axis cine images at $1.5 \mathrm{~T}$ were obtained following adenosine stress and gadolinium contrast, whereas short axis images at $3 \mathrm{~T}$ and all long axis images were acquired before contrast administration. Scans were analysed offline by a single experienced observer blinded to patient details, using cmr42 software (Circle Cardiovascular Imaging, Calgary, Canada). End-diastolic epicardial and endocardial and end-systolic endocardial borders were defined manually and propagated by the software throughout the cardiac cycle to derive longitudinal, circumferential and radial global systolic strain (GLS, GCS and GRS) and peak early diastolic strain rates (longitudinal, circumferential and radial PEDSR). Inter-field strength
Abstract 10 Table 1 Left ventricular function, volume and strain measurements

\begin{tabular}{llll}
\hline LV function and volume & & \\
\hline LVEF (\%) & $59.8(4.1)$ & & \\
LVEDV (ml) & $158.6(28.1)$ & & \\
LVESV (ml) & $64(14.1)$ & & \\
LV mass (g) & $87.1(21)$ & & ICC \\
LV strain measurements & $1.5 T$ & $3 T$ & 0 \\
GLS (\%) & $-17.5(1.4)$ & $-15.6(1.4)$ & 0.49 \\
GCS (\%) & $-19.8(2)$ & $-18.4(2.3)$ & 0.49 \\
GRS (\%) & $35.3(6.1)$ & $31.7(6.7)$ & 0.49 \\
Longitudinal PEDSR $\left(\mathrm{s}^{-1}\right)$ & $0.87(0.14)$ & $0.72(0.1)$ & 0 \\
Circumferential PEDSR $\left(\mathrm{s}^{-1}\right)$ & $1.03(0.14)$ & $0.93(0.21)$ & 0.3 \\
Radial PEDSR $\left(\mathrm{s}^{-1}\right)$ & $-2.06(0.42)$ & $-1.76(0.56)$ & 0.12 \\
\hline
\end{tabular}

mean (SD): LV, left ventricle; LVEF, left ventricular ejection fraction, LVEDV, left ventricula end diastolic volume; LVESV, left ventricular end systolic volume; GLS, global longitudinal strain; GCS, global circumferential strain GRS, global radial strain; PEDSR, peak end diastolic strain rate; ICC, intraclass correlation coefficient

agreement was assessed with intraclass correlation (ICC) and Bland Altman plots.

Results Mean age was $50 \pm 6.3$ years and 50\% were male. LV function, volumetric and strain data are presented in table 1. Mean systolic strain measurements were higher at $1.5 \mathrm{~T}$ versus 3T (all $\mathrm{p}<0.05$ ). Inter-field strength agreement was fair for GCS and GRS (ICC=0.49). Despite all images being acquired before contrast, there was no agreement for GLS (ICC $=0$; table 1). Bland Altman plots are shown in figure 1. All PEDSR measurements had poor agreement (ICC range $0-0.3$; table 1).

Conclusion In this pilot study, strain measurements showed fair inter-field strength agreement for GCS and GRS and no agreement for GLS. This may relate to different signal-to-noise characteristics between field strengths. Further work is needed with randomised comparisons, but these data suggest strain measurements obtained at $1.5 \mathrm{~T}$ and $3 \mathrm{~T}$ are not interchangeable.

\section{NOVICE MARATHON TRAINING REVERSES VASCULAR AGEING}

${ }^{1,2} \mathrm{AN}$ Bhuva, ${ }^{3} \mathrm{~A}$ D'Silva, ${ }^{2,4} \mathrm{C}$ Torlasco, ${ }^{1} \mathrm{~S}$ Jones, ${ }^{1} \mathrm{~N}$ Nadarajan, ${ }^{2} \mathrm{~J}$ Van Zalen, ${ }^{2} \mathrm{R}$ Boubertakh, ${ }^{1} \mathrm{~N}$ Chaturvedi, ${ }^{2} \mathrm{G}$ Lloyd, ${ }^{3} \mathrm{~S}$ Sharma, ${ }^{1,2} \mathrm{~J} \mathrm{C}$ Moon, ${ }^{1} \mathrm{AD}$ Hughes, ${ }^{1,2} \mathrm{CH}$ Manisty. 'Institute for Cardiovascular Science, University College London, London, UKi ${ }^{2}$ Department of Cardiovascular Imaging, Barts Heart Centre, Barts Health NHS Trust, London, UK; ${ }^{3}$ Cardiology Clinical and Academic Group, St George's, University of London, London, UK; ${ }^{4}$ Istituto Auxologico Italiano, IRCCS, Dept of Cardiovascular, Neural and Metabolic Sciences, San Luca Hospital, Italy

\subsection{6/heartjnl-2019-BSCMR.11}
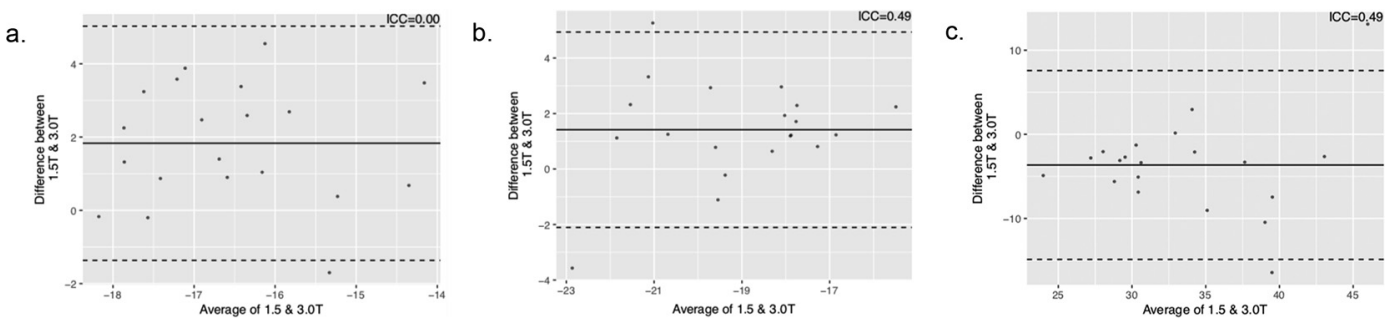

Abstract 10 Figure 1 Bland Altman plots for systolic strain measurements; a. global longitudinal strain (GLS), b. global circumferential strain (GCS), c. global radial strain (GRS) 